\title{
Décrire des objets hybrides
}

Les grandes ignames décorées du village de Nyamikum, province de l'Est Sepik, Papouasie-Nouvelle-Guinée

How to Describe Hybrid Objects

\section{Ludovic Coupaye}

\section{(2) OpenEdition}

12 Journals

Édition électronique

URL : https://journals.openedition.org/tc/4730

DOI : $10.4000 /$ tc. 4730

ISSN : 1952-420X

Éditeur

Éditions de l'EHESS

\section{Édition imprimée}

Date de publication : 1 décembre 2009

Pagination : 50-67

ISBN : 978-2-7351-1301-9

ISSN : 0248-6016

\section{Référence électronique}

Ludovic Coupaye, « Décrire des objets hybrides », Techniques \& Culture [En ligne], 52-53 | 2009, mis en ligne le 19 juillet 2012, consulté le 29 septembre 2022. URL : http://journals.openedition.org/tc/4730 ; DOI : https://doi.org/10.4000/tc.4730 


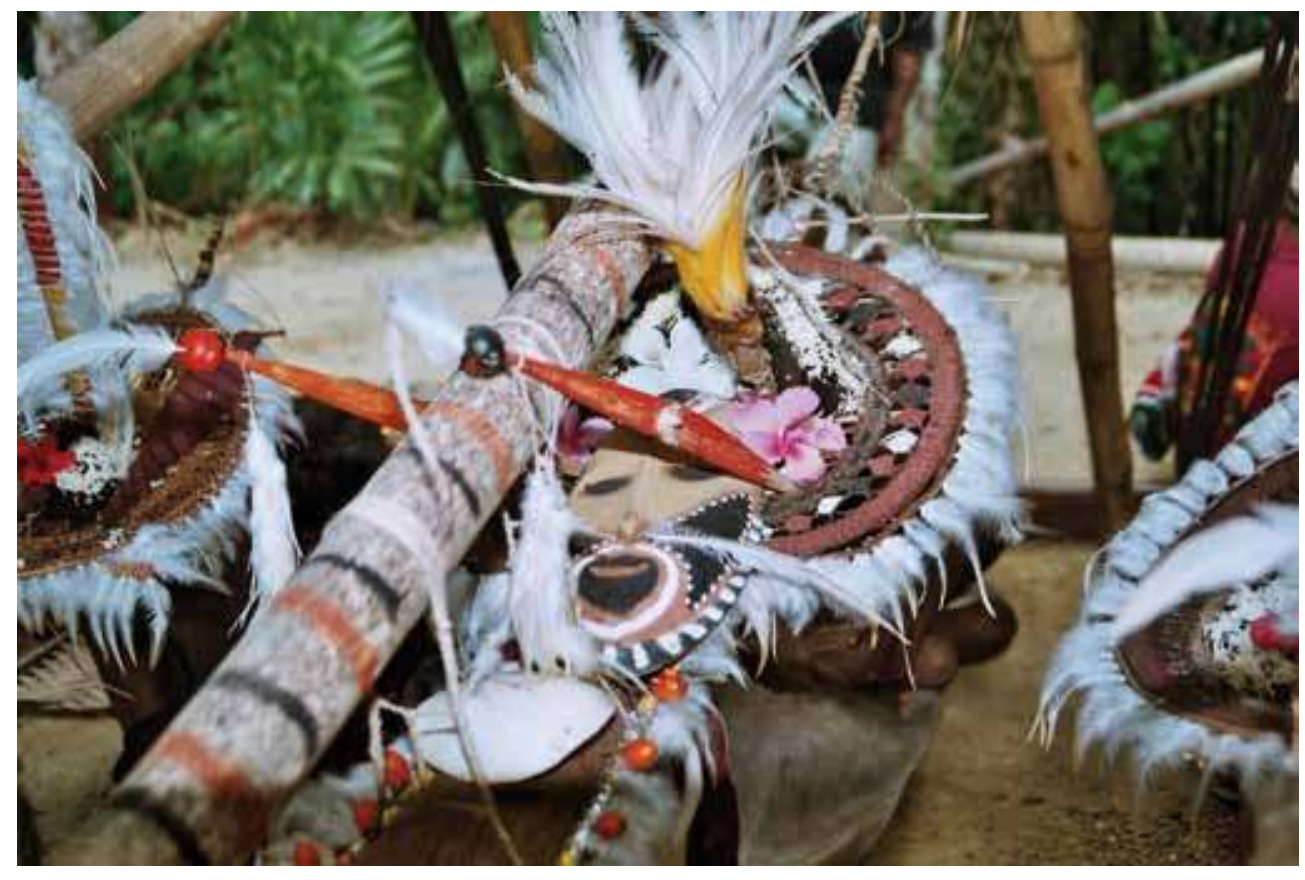




\title{
DÉCRIRE DES OBJETS HYBRIDES
}

\section{Les grandes ignames décorées du village de Nyamikum, province de l'Est Sepik, Papouasie-Nouvelle-Guinée}

\author{
Où l'on verra comment la chaîne opératoire est un instrument idéal pour révéler \\ les connections entre matérialité et idéalité.
}

L'objectif principal est d'illustrer brièvement des éléments théoriques et méthodologiques issus des approches actuelles de l'anthropologie de l'art et de la culture matérielle. Pour les besoins de la démonstration, nous faisons appel à une combinaison inédite d'éléments née des approches britanniques de la culture matérielle (cf. Coupaye \& Douny, ce numéro), de l'anthropologie de la Mélanésie (e.g. Jeudy-Ballini \& Juillérat 2002; Strathern 1999; Wagner 1986, 1991) et de la technologie culturelle (Cresswell 1996). Les grandes ignames (Dioscorea alata) des Abelam sont célèbres dans la littérature anthropologique océanienne (Kaberry 1941; Lea 1961; Tuzin 1972; Rubel \& Roseman 1978; Hauser Huber-Greub 1988). Cultivés sur essarts exclusivement par les hommes, ces tubercules peuvent atteindre trois mètres de long et peser plus de cinquante kilogrammes pour le cultivar considéré comme le plus important, la Maambutap.

Une fois récoltées, les ignames sont décorées et présentées lors de cérémonies annuelles, appelées à Nyamikum Waapi Saaki (« alignement des grandes ignames ») qui clôturent le cycle des récoltes (Fig. 1-2). L'ensemble des habitants des différents hameaux du village, ainsi que des visiteurs des villages voisins sont invités à les évaluer (Fig. 3-4). Les grandes ignames entrent ensuite dans des circuits d'échanges complexes comprenant entre autres les échanges compétitifs entre des partenaires cérémoniels, les 
Cérémonie Waapi Saaki « Alignement des grandes ignames » à Balukwil-Nyamikum (août 2003) Les ignames waapi (Dioscorea alata) de la variété locale Maambutap sont présentées pour évaluation. Le public est constitué des gens des hameaux du village ainsi que de délégations venues des villages voisins, alliés comme rivaux. Il existait autrefois trois cérémonies successives, chacune consacrée à un groupe de variétés spécifiques. Chaque cérémonie se décompose en une présentation des cultivars, suivie de leur évaluation, de discours publics puis d'une danse qui va durer toute la nuit.

(Fig. 1) compensations matrimoniales et funéraires et le règlement de disputes. D’une façon générale, une fois le tubercule transmis, une partie en est consommée par son acquéreur et ses alliés, tandis que l'autre sert de bouture pour être replantée ${ }^{1}$.

Les habitants cultivent également des petites ignames (Dioscorea esculenta) et un ensemble d'autres plantes associées, comme le taro, une variété comestible d'ambrette (Abelmoschus manihot), et plusieurs variétés de
Waapi Saaki, Nyamikum (juin 2003) Deux Grands Hommes venus d'un village voisin évaluent une grande igname de la variété kupmi. (Fig. 3)
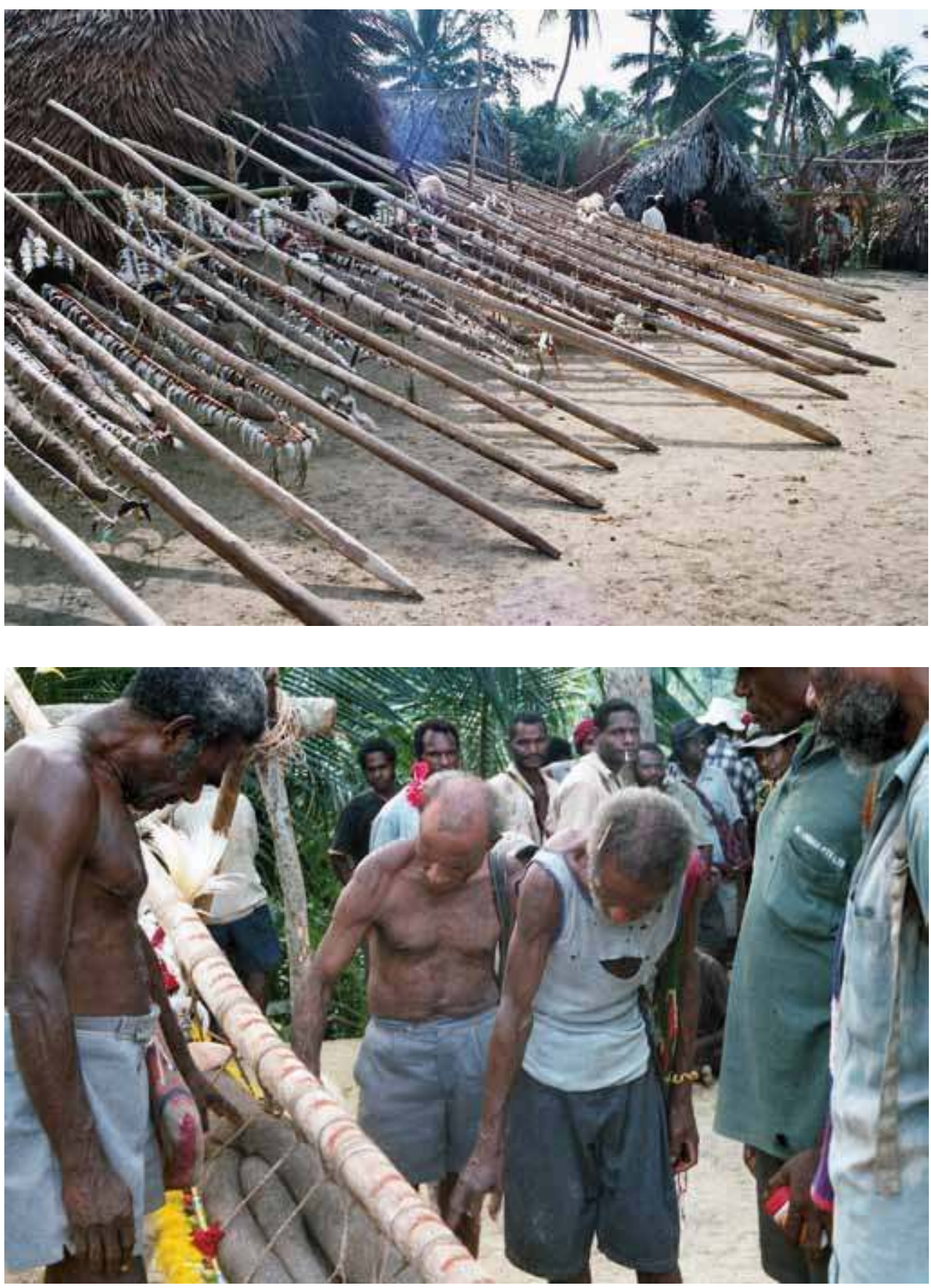
bananes. Les grandes ignames, quant à elles, sont cultivées dans d'autres jardins, isolés et généralement clôturés, dont l'accès est interdit aux femmes et aux étrangers (Fig. 5-6). En revanche, les petites ignames sont plantées en commun et cultivées dans des jardins ouverts où hommes et femmes travaillent de concert (Fig. 7).

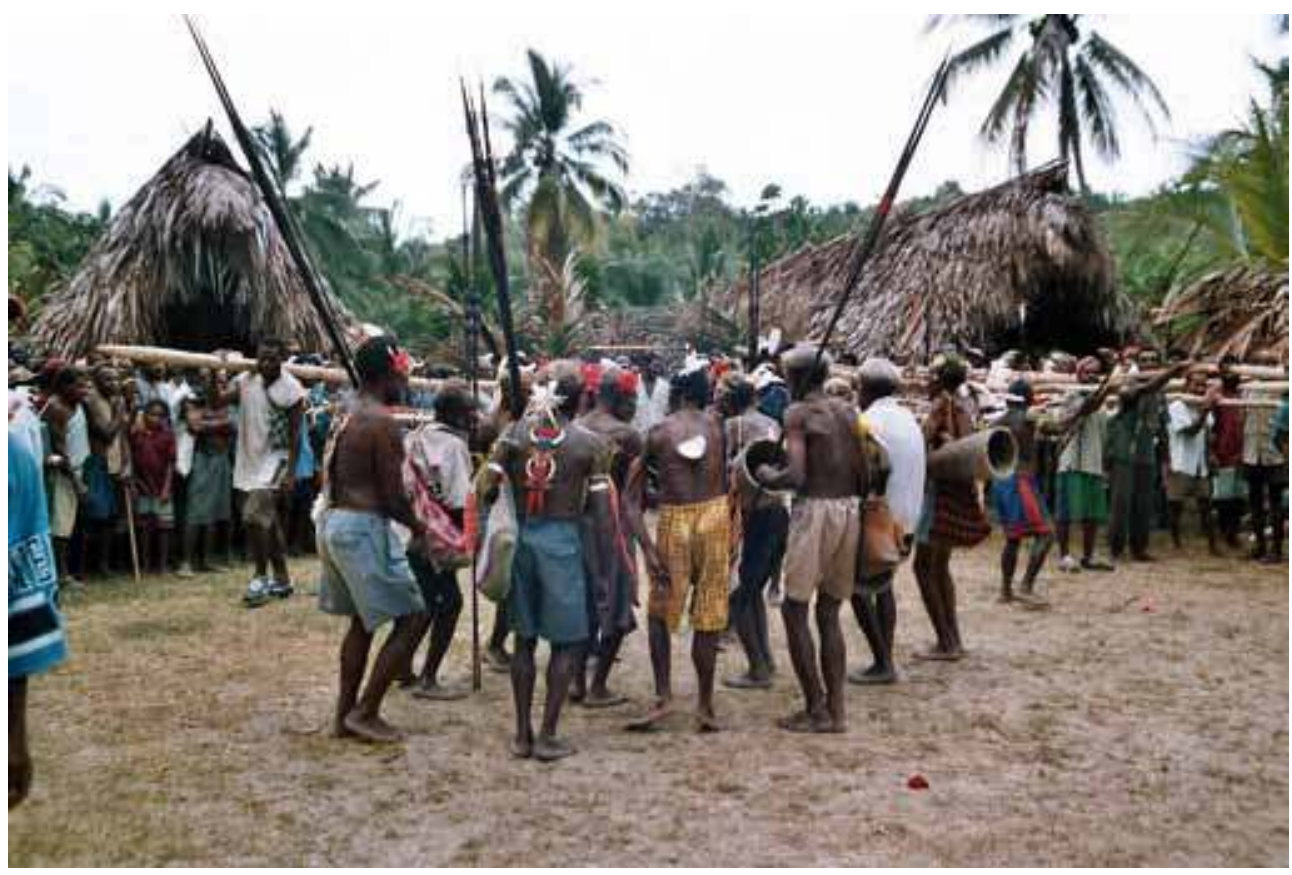

Waapi Saaki à Kimbangwa

(juillet 2003)

Au début de la cérémonie, les ignames sont apportées sur la place cérémonielle, précédées par un groupe de Grands Hommes, chantant en s'accompagnant de tambours-sabliers.

Chaque igname, portée par deux

hommes, fait le tour de la place avant d'être installée sur une des structures prévues (cf. Fig. 1). (Fig. 2)

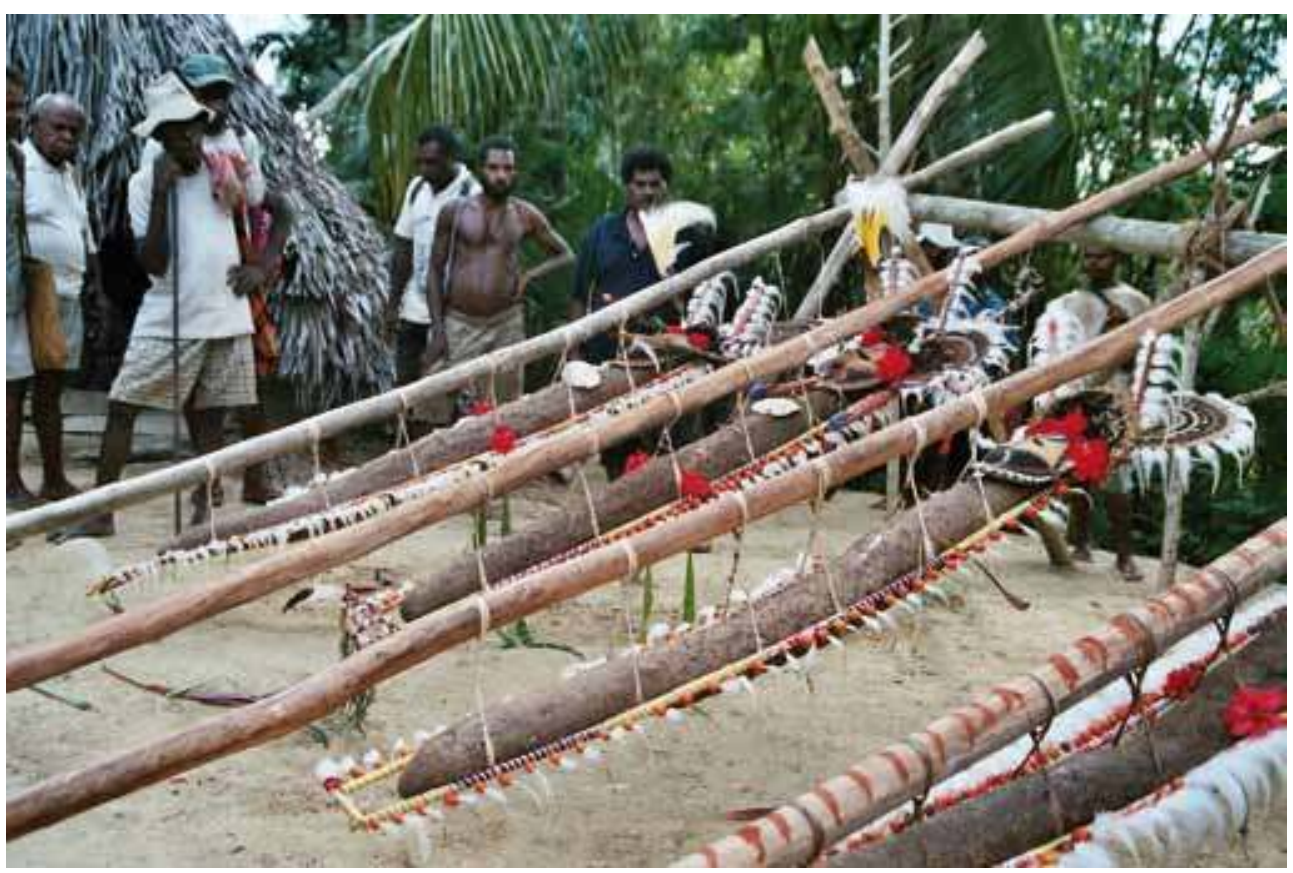

Waapi Saaki, Nyamikum (juin 2003)

Les ignames présentées sur cette photo (des Maambutap), sont suspendues à leurs montants, et reposent sur la structure construite sur la place cérémonielle.

On distingue une partie des décorations, comme le masque de bois, l'ornement fait d'une dépouille de Paradisier (traitée pour qu'elle puisse se conserver), de coquillages et de plumes. (Fig. 4) 


\section{Un objet complexe et multivalent}

Les grandes ignames sont au cœur d'un ensemble de caractéristiques matérielles, conceptuelles et symboliques qui les placent à l'intersection de plusieurs registres sociaux: nourriture, monnaies cérémonielles et images d'ancêtres. Comme nourriture, leur consommation contribue à la constitution de la personne physique, mais aussi sociale. En tant que

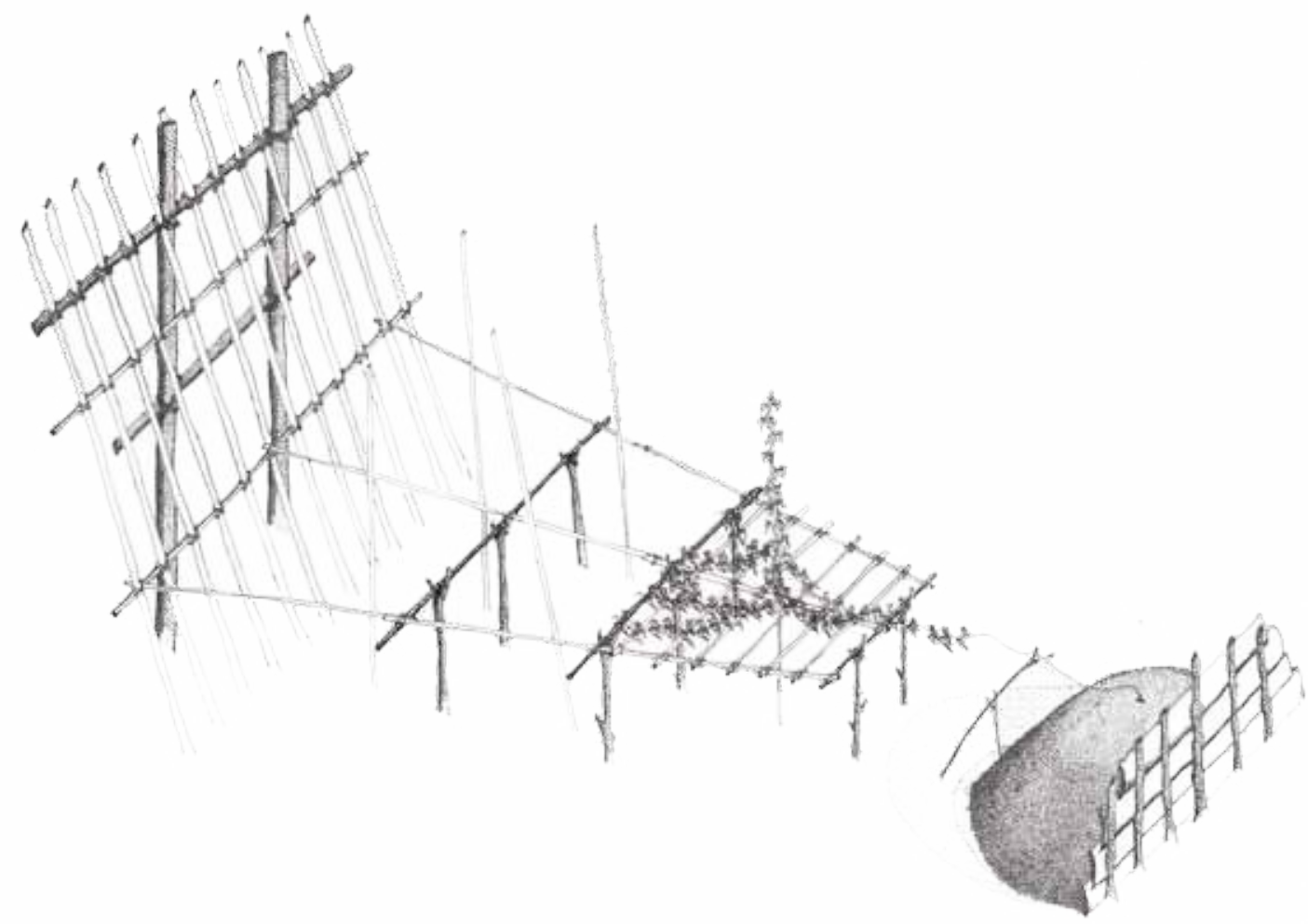

Vue axonométrique de la treille à grande igname

Cette treille se compose des éléments suivants: tëkët (le « siège ») soutenant (le monticule) kutapmë; jaabë (le « lit » horizontal) sur lequel vont reposer les tiges de la plante et taawu (la « structure » en verticale) sur laquelle vont grimper les tiges de la plante au terme de leur croissance.

(Fig. 5) - Dessin de l'auteur. 


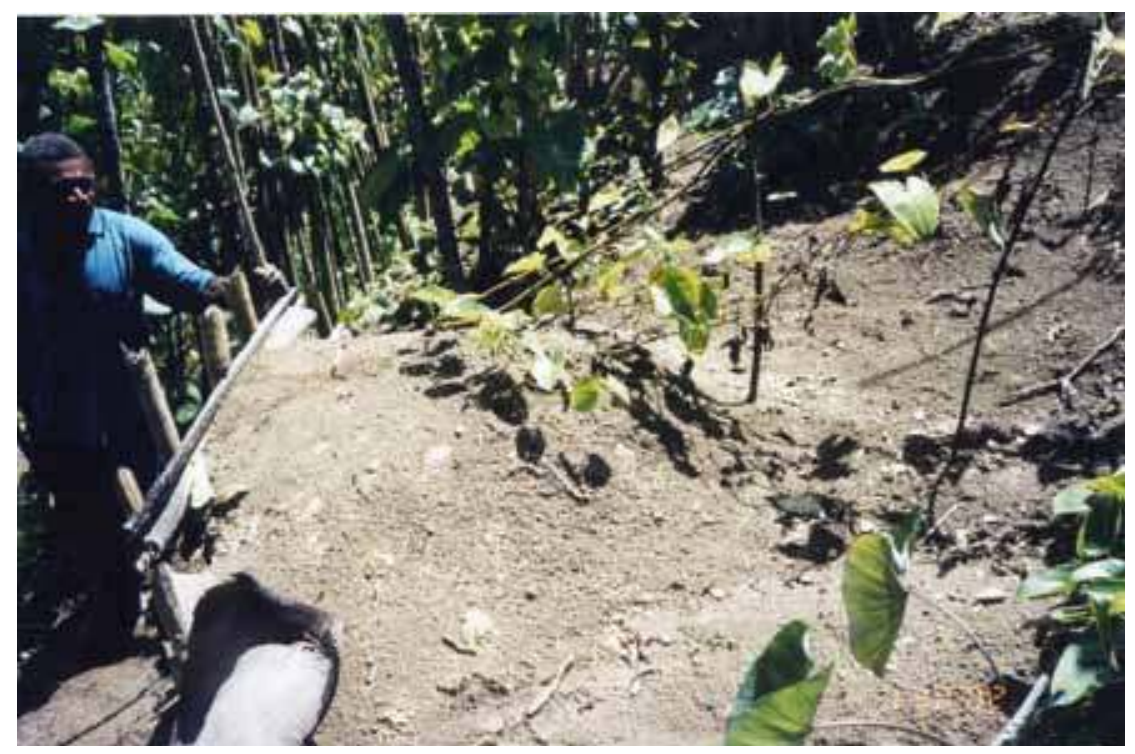

Jardin à Igname de Kony Ganbakiya

(février 2002)

Vue du monticule: kutampë.

(Fig. 6)

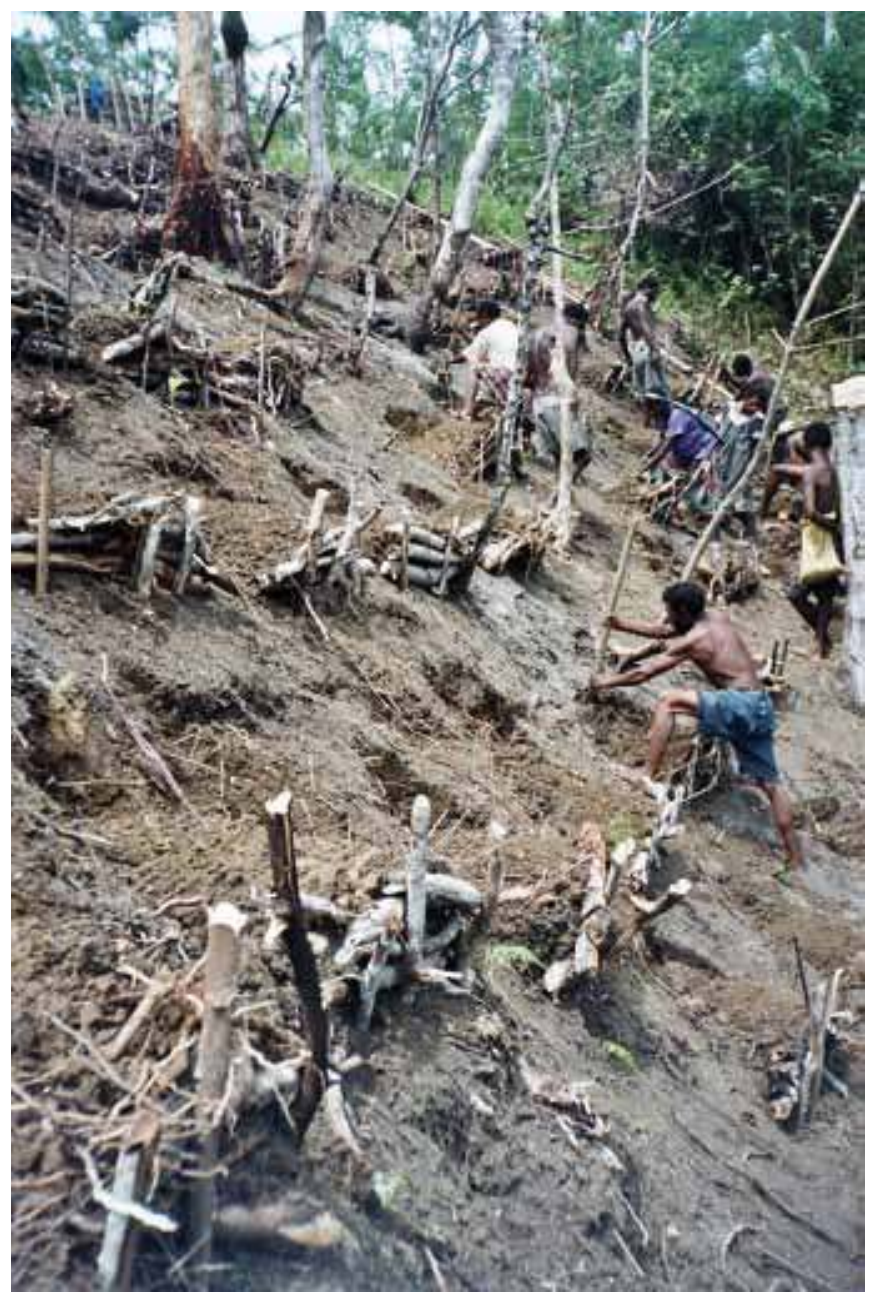

Plantation d'un jardin de petites ignames $k \boldsymbol{b}$

Contrairement à la plantation des grandes ignames, celle d'un jardin de $k a$ mobilise l'ensemble du hameau. Les jeunes gens manient les bêches en palmier noir (gësad), et préparent pour chaque emplacement à igname, un mini tëkët (cf. figure 5 et 6 ), destiné à soutenir le petit monticule. Hommes et femmes installent les tubercules dans leurs trous, avant de les recouvrir. (Fig. 7) 


\section{Danseurs du village de Nyamikum (mai 2002)}

Pour la cérémonie célébrant le début du revêtement de la route reliant Maprik à Wewakn, capitale de la province, les danseurs sont décorés comme l'étaient les initiés à l'occasion de la cérémonie de clôture des initiations, aujourd'hui abandonnées. (Fig. 8) monnaies cérémonielles, elles jouent le rôle de substituts d'êtres humains dans les systèmes de compensations. Leur échange contribue au maintien et à la reproduction des relations sociales, en faisant circuler les boutures à l'intérieur et à l'extérieur du village. Enfin, par leur révélation lors de cérémonies, elles évoquent d'autres formes symboliques absentes et invisibles comme celles des ancêtres, renvoient également aux pouvoirs invisibles qui animent les espèces vivantes et contribuent à leur croissance et à leur reproduction. En tant que telles, elles sont décorées comme l'étaient autrefois les initiés (Fig. 8) et présentent des connotations visuelles qui les rapprochent des images d'entités ancestrales peintes sur les façades des maisons cérémonielles (Fig. 10) ou des figures sculptées et polychromes, qui étaient révélées lors des cycles d'initiations (Forge 1973) aujourd'hui abandonnés. Les éléments de leur décoration, notamment les masques en vannerie, ont aujourd'hui pénétré le domaine du marché international de l'art et font partie des objets emblématiques des productions Abelam dans les collections publiques comme privées (Fig. 9).

Ainsi, les grandes ignames décorées apparaissent comme des objets complexes et multivalents dont l'étude est l'exemple privilégié d'un ensemble de phénomènes étudiés aujourd'hui en anthropologie de la Mélanésie (cf. Jeudy-Ballini et Juillerat 2002), parmi lesquels on peut mentionner les échanges, les rapports hommes-femmes, le foncier, l'agriculture traditionnelle, la personne sociale, les rituels, l'art et les phénomènes esthétiques, ou encore la modernité.

La question reste de savoir comment ces différents phénomènes interviennent, sont incorporés et concrétisés dans cet objet. De comprendre pourquoi cet élément végétal est si essentiel aux yeux des gens de Nyamikum et les placent à part des autres productions végétales.

\section{La méthode d'approche}

Les Abelam étant connus pour l'absence quasitotale d'interprétations élaborées, confirmant le caractère non-verbal de ce qui est transmis durant les cérémonies (Forge 1973), il est difficile de recourir à la solution des exégèses locales pour répondre à cette question. Par ailleurs, les explications classiques sont souvent limitées aux qualificatifs de « cérémoniel » ou de « rituel » (Tuzin 1972). Elles ne suffisent pas à rendre 


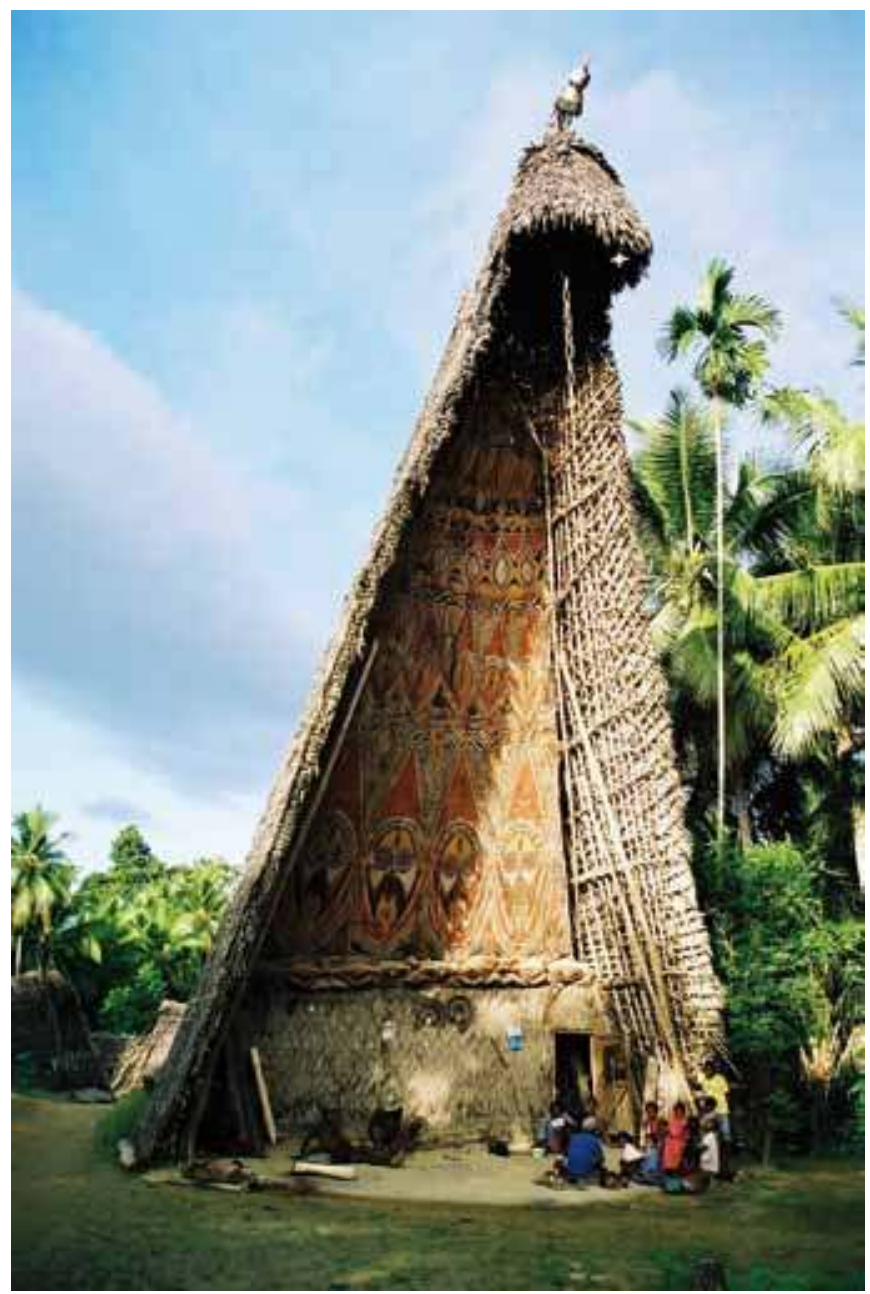

Maison cérémonielle du village de Kimbangwa

(décembre 2001)

(cf. Huber-Greub 1986)

La façade présente, en registre, des images des entités totémiques des clans (nGwaa). On notera que ces figures évoquent celles des masques à ignames en bois ( $c f$. Fig. 8 et 9 ).

(Fig. 10)

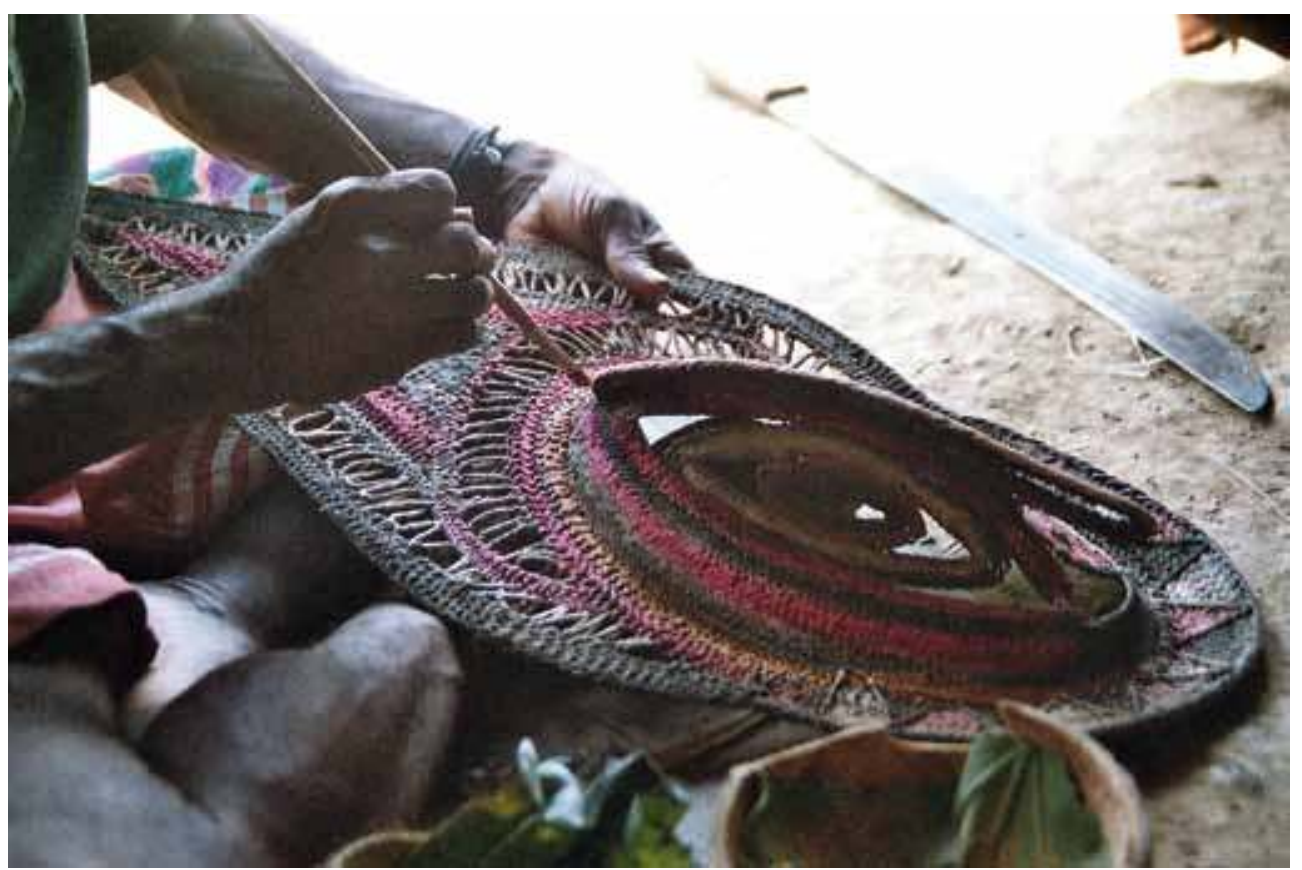

Balukwil

(août 2003)

Préparation d'un masque à grande igname en fibres.

(Fig. 9) 
compte du rôle concret que de tels objets jouent ou de ce qui leur donne leur valeur, en dehors d'éléments qui sont jugés remarquables - tels l'isolement des jardins, le caractère exclusivement masculin et la série d'interdits alimentaires et comportementaux, notamment sexuels, qui sont associés au tuberculeartefact et qui le distinguent des autres productions horticoles.

Face à une telle situation, l'approche adoptée pour répondre à la question du « comment » a été d'analyser en détail la chaîne opératoire de production conduisant à la fabrication des ignames en tant qu'artefacts originaux. C'est par ce biais que l'on a choisi aussi d'éclairer un certain nombre de concepts et de notions qui prédominent dans les études anthropologiques contemporaines de la culture matérielle et notamment dans les approches qui sont au cœur des études anglo-saxonnes (cf. Coupaye\& Douny, ce numéro). Nous n'en citerons ici qu'un nombre restreint à titre d'exemple.

Ce choix méthodologique permet de « donner matière » à des concepts tels que la « biographie des objets » (Kopytoff 1986), la « matérialité » (terme renvoyant au caractère relationnel des propriétés matérielles et sensorielles de l'objet (cf. Miller 2005), l'agency (Gell 2008 [1998])² et de comprendre comment l'usage et la consommation des objets (Miller 1987) participent à la co-construction du social et des corps (Warnier 1999). Au passage, on notera que cette approche via la chaîne opératoire permet aussi de suivre la proposition de Bruno Latour (1991) et d'étudier un objet « hybride », ici un parfait mélange de nature et de culture, en étudiant l'enchevêtrement des deux pôles au lieu de chercher à les distinguer. La chaîne opératoire est également l'occasion de mettre conjointement en relief les actions sur la matière et l'ensemble des « réseaux » d'acteurs humains et non-humains mobilisés dans ces opérations. Enfin, il devient possible de comprendre comment la monstration de tels objets complexes et multivalents, par les inférences esthétiques et matérielles qu'ils génèrent chez leurs spectateurs ou par affordance (Gibson 1977) est à la source d'une forme « d'enchantement » dont le rôle est de transformer ceux qui en sont les témoins (Gell 1992). Ces notions et leur validité sont bien sûr toujours soumises à débat (par exemple voir Ingold 2007 ; Bloch 1999). Il nous semble cependant que leur combinaison dans l'approche proposée permet de les éclairer.

Par conséquent, nous avons opté pour une attitude sensiblement différente des approches de la technologie culturelle tout en conservant ses principes. Nous avons choisi d'examiner non seulement les actions sur la matière, mais également celles considérées comme rituelles, et plus encore, l'ensemble des « réseaux » d'acteurs humains et non-humains mobilisés dans ces opérations. Non seulement il s'agissait de suivre les remarques de Mauss sur l'efficacité, commune aux actes techniques et aux actes rituels (1968 [1909], 1950 [1935]), mais également de dépasser la question des déterminismes sociaux et/ou matériels, en remplaçant la recherche des causes par celles des raisons données par les cultivateurs eux-mêmes, au sens des « nécessités logiques» (Wittgenstein 1993 [1922] ; de Lara 2005). 


\section{La chaîne opératoire comme réseau complexe}

Le processus de production des ignames ${ }^{3}$ se compose d'une grande variété de chaînes opératoires emboîtées les unes dans les autres ${ }^{4}$, que l'on peut résumer comme suit:

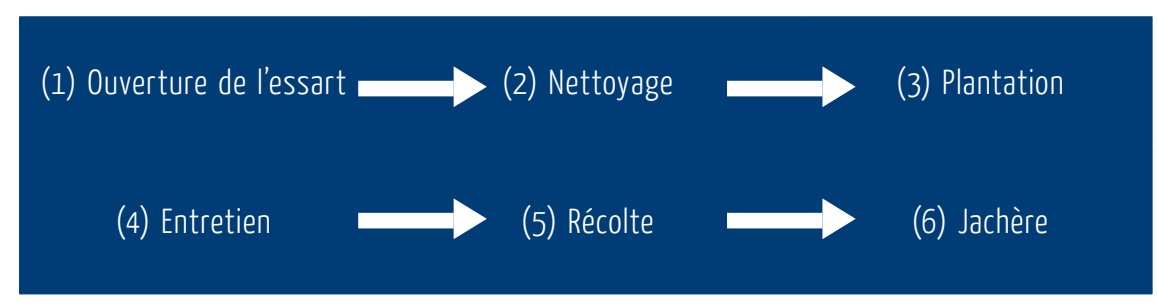

Chacune de ces étapes peut être décomposée en une multitude d'autres étapes, certaines prenant quelques minutes, d'autres s'étendant sur une période pouvant aller jusqu'à plusieurs mois. On notera, qu'une récolte (étape 5) peut être suivie d'une nouvelle plantation (retour à l'étape 3), et ce jusqu'à deux ou trois cycles avant de laisser la forêt avoisinante reconquérir l'espace du jardin. Cette séquence est valide pour les jardins des grandes comme des petites ignames ${ }^{5}$

Toutefois, la séquence décrite précédemment ne prend pas en compte la cérémonie Waapi Saaki qui clôture un cycle agricole. La raison pour laquelle les cultivateurs investissent autant de temps et d'énergie dans la culture d'un seul tubercule (Fig. 11-12) ${ }^{6}$ parallèlement à la culture d'un jardin commun qui en produira plus de 1300 pour un poids total approximatif de $1100 \mathrm{~kg}$, est exprimée bien souvent par les Abelam de la manière suivante:

« si nous ne récoltons pas de Maambutap et si nous ne faisons pas de cérémonie, aucune nourriture ne pourra sortir des jardins et nous mourrons de faim. Les Maambutap "ouvrent la route" à toute la nourriture ».

Cette remarque est importante pour la compréhension du phénomène. En effet, elle permet à elle seule de justifier l'inclusion du Waapi Saaki dans la chaîne opératoire globale comme étape nécessaire à la production de nourriture.

La séquence précédente doit donc être articulée à la seconde afin d'intégrer les dimensions symboliques du processus:

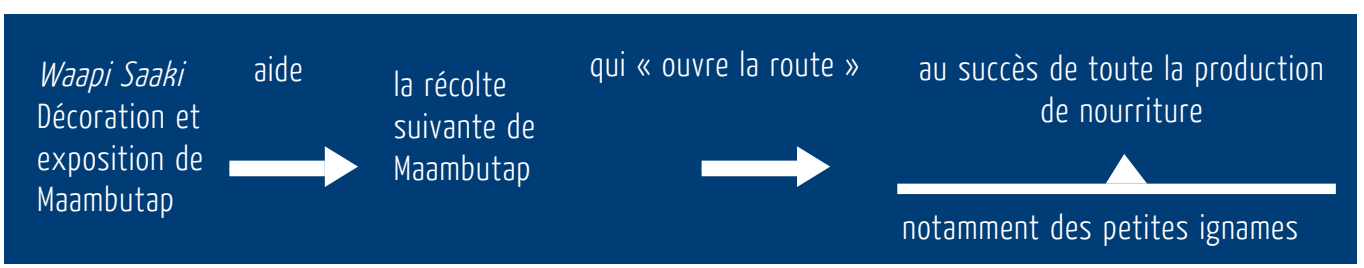




\section{Bill Kasëpëk récoltant une Maambutap}

Après avoir démonté une partie du tëkët (cf. Fig. 5). il gratte la terre entourant le tubercule à l'aide d'un gwalpany (outil confectionné à partir d'un fémur de cochon).

(Fig. 11)
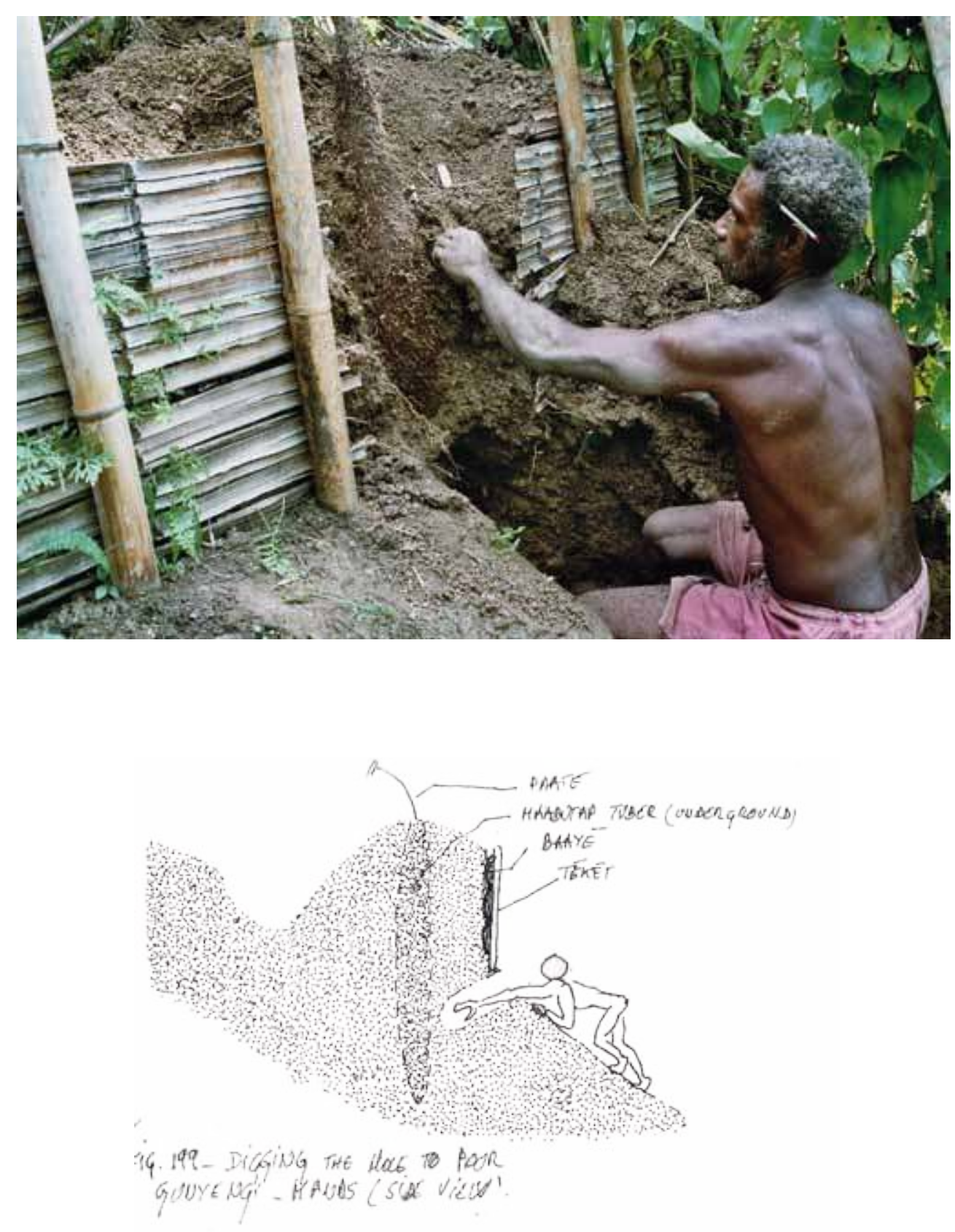

\section{Croquis de terrain}

II montre la position d'un homme creusant un tunnel pour accéder à la partie inférieure de l'igname. En fonction du niveau auquel il arrive, le cultivateur peut descendre un peu plus bas afin d'atteindre l'extrémité du tubercule. Puis, en fonction de son évaluation de la pousse, il peut décider de rajouter des " fertilisants », composée de sève et/ou de poudres tirées de pierres ou de cendres. (Fig. 12) 
Les discussions avec les cultivateurs font également ressortir d'autres éléments jugés essentiels à la réussite de l'ensemble des opérations. Pour des raisons d'espace, nous n'en esquisserons que quelques-uns:

- La nécessité de planter ses ignames sur la terre clanique, qui est censée reconnaître les siens, ou d'obtenir l'accord d'un représentant du clan auquel la terre appartient, accord sanctionné par un rituel.

- La qualité du Jëwaai, une substance corporelle invisible qui varie selon les individus (certains lignages sont considérés comme plus favorisés que d'autres), joue un rôle central notamment mais pas exclusivement dans la capacité à faire pousser des plantes; la nécessité d'observer pendant toute la durée de la pousse des grandes ignames, qui s'étend sur plus de huit mois, un Yakët. Ce terme regroupe une série de prescriptions et de proscriptions alimentaires et comportementales. Ces dernières incluent notamment des interdits sexuels et la nécessité d'éviter tout conflit, dispute ou colère qui ont un effet négatif sur la pousse des tubercules. Ainsi, autrefois, les guerres avec les villages voisins étaient suspendues. Par ailleurs, les interdits sexuels évitent au corps du cultivateur d'accumuler des substances issues du sang menstruel féminin, considéré comme puissant mais néfaste pour la plupart des activités physiques et rituelles, et susceptible d'affecter la forme finale des tubercules, qui doivent être longs, réguliers et sans fourches ni bulbes. Parmi les prescriptions, on notera la nécessité de réaliser une saignée du pénis, opération douloureuse, mais jugée nécessaire afin de débarrasser le corps des substances néfastes.

- Le rôle des femmes, et notamment des épouses, est également impliqué dans l'observation parallèle d'un Yakët destiné à modifier aussi leur corps. Celui-ci se justifie par le fait que l'épouse nourrit le cultivateur durant toute la durée du cycle, et qu'elle l'accompagne dans une grande partie des tâches quotidiennes. Dans certains cas, si le Jëwaai de l'épouse est bon, si elle respecte les interdits sexuels et si elle n'est pas en période de règles, elle peut accompagner le cultivateur au jardin de grandes ignames. Bien qu'il s'agisse d'exceptions, ceci invite à penser que ce ne sont pas les femmes ni les éléments féminins du monde qui sont exclus, mais uniquement ce qui touche à la menstruation. Ainsi, les filles prépubères (plus encore que les jeunes garçons) ou les femmes ménopausées peuvent être préférées pour la réalisation d'opérations rituelles à l'intérieur du jardin, si l'on peut être certain, dans le premier cas, qu'elles n'ont ni menstruations, ni pu avoir de rapports sexuels, et dans le second, celui des femmes ménopausées, qu'elles ne possèdent plus cette substance dangereuse.

- Le recours à un ensemble de rites (privés et individuels) réalisés avant d'entrer dans le jardin ou à l'intérieur du jardin. Ils incluent des purifications corporelles destinées à « aiguiser » le Jëwaai ou à mobiliser l'assistance d'entités totémiques non-humaines du clan du cultivateur, résidant dans des portions identifiées 
du territoire clanique et dont l'action est relayée par des vers de terres invisibles. Ces derniers contrôlent la pousse des tubercules et peuvent sentir le Jëwaai de l'individu par l'intermédiaire du contact des mains avec la terre ou de la sueur tombant sur le sol. Ces entités sont mobilisées par l'entremise de formules énoncées ou simplement murmurées, voire pensées. Ces deux ensembles de rites non seulement agissent sur ces entités, mais améliorent également les qualités corporelles de l'individu, aiguisent ses sens et donnent leur efficacité aux interactions gestuelles avec les matériaux manipulés, particulièrement avec la terre et les vignes grimpantes des ignames.

- L'ajout de substances magiques (cf. Forge 1962) lors de la croissance du tubercule. Ces substances sont soit récoltées par le cultivateur lui-même soit obtenues par échanges entre alliés et parents audelà des frontières du village. Ces substances peuvent être des poudres minérales (de valence mâle) ou des décoctions liquides (de valence femelle), ou encore des combinaisons des deux. Elles ont pour origine des lieux particuliers du territoire, lieux réputés pour être les domaines d'entités non-humaines potentiellement dangereuses de sexe masculin ou féminin. Chaque cultivateur possède sa propre recette, soit une création personnelle, à partir d'expérimentations, de conseils ou d'opinions soit obtenue par échanges.

- Le dépôt d'un anneau de coquillages auprès d'un autel secret contenant une pierre sacrée considérée comme la source du pouvoir procréatif des Maambutap, alimentait « comme une centrale électrique », selon les dires des habitants de Nyamikum, tous les jardins appartenant aux individus ayant déposé un de leurs anneaux. Chaque village possède un jeu de pierres particulier, chacun dédié à un type de nourriture spécifique (telle que grandes ignames, petites ignames ou arbres à pain). Tous les villages possèdent une pierre dédiée aux Maambutap. En fonction du succès d'un village, celui-ci peut recevoir des demandes de la part de cultivateurs extérieurs.

- Les interactions sociales jouent aussi un rôle important. Non seulement les cultivateurs sont censés adopter une attitude permanente de Nëmandu (Grand Homme) mais ils doivent également participer activement aux débats publics ayant lieu sur les places cérémonielles au centre desquelles se trouve un petit amas de pierres appelé Baapmu-taakwa. Ces débats ont lieu régulièrement, lors des réunions d'information bimensuelles du village sous l'égide du Conseiller, représentant du village à la Chambre du District de Maprik, ou à la clôture de travaux réalisés en communs (tels que la construction d'une maison de réunion au sein d'un hameau, ou la plantation d'un jardin), de funérailles ou, bien sûr, d'un Waapi Saaki. Ces discours et débats, animés, utilisent un mode métaphorique. Ils sont censés « chauffer » la Baapmu-taakwa, et 
cette chaleur est retransmise à la pierre sacrée cachée mentionnée plus haut qui la conserve. Les relations sociales de l'ensemble du village sont donc à la source même du pouvoir de reproduction des pierres.

\section{$\&$}

L'ensemble des chaînes opératoires impliquées dans la culture des grandes ignames mobilise un ensemble hétérogène de gestes, d'actions sur la matière et le corps, de substances, d'acteurs, de lieux et de temporalités (Fig. 13-14-15) qui ont tous une raison d'être spécifique et sont mobilisés pour atteindre un résultat efficace. L'objet fini est donc un condensé de relations (sociales, matérielles, symboliques) réalisées au cours de la chaîne opératoire et concrétisées sous la forme d'un seul artefact (Fig. 16) qui doit impérativement être donné à voir. La récolte d'une Maambutap digne d'être présentée est vue comme l'assurance de la production de nourriture pour l'ensemble du village.

Du point de vue des concepts évoqués plus haut, la matérialité de l'objet ne s'arrête donc pas aux qualités passives, qu'elles soient physiques, sensorielles ou symboliques du tubercule, lesquelles ne seraient révélées que par les usages, quand bien même multiples (comme nourriture, bouture, monnaie ou image) que l'on peut en faire. Elle s'étend aussi aux propriétés engendrées par la multitude d'agents, certains en opposition et en contradiction, mobilisés et intégrés lors des opérations techniques que cet objet concrétise et restitue au moment de sa monstration. Cette multivalence en fait un objet composite qui contient une partie de la personne du cultivateur et qui peut à son tour créer de nouvelles relations/tubercules. Cet objet peut-être interprété comme un microcosme. Il contient le potentiel de reproduction d'autres ignames, mais évoque aussi les propriétés d'autres images absentes (figures d'ancêtres, initiés) et dont la monstration génère, par inférences, d'autres images possibles (métaphores visuelles et verbales: pénis, sculpture, corps humain), tout en les fixant (temporairement) dans l'objet-igname lui-même. L'igname donne à voir les relations déjà réalisées mais aussi potentielles (qui se manifesteront lors de la consommation, de la plantation ou des échanges) sans rien dévoiler du processus technique dont elle est la concrétisation.

Évalué par d'autres cultivateurs, le tubercule suscite des inférences à la fois verbales et non verbales, non seulement sur sa forme, sa taille, sa couleur ou la régularité de sa
Westley Kitnyora utlisant un gësaa (« bâton à fouir ») (novembre 2002)

Il permet de préparer le trou pour planter une petite igname $(k a)$ durant une session collective de plantation d'un jardin. Placé de profil par rapport à la direction du gësaa, d'une percussion lancée, il plante la pointe de l'outil, avant d'appuyer sur le manche, pour faire levier et soulever la terre. Ce travail, accompli par les jeunes gens est souvent accompagné de plaisanteries et d'allusions sexuelles.

(Fig. 13)

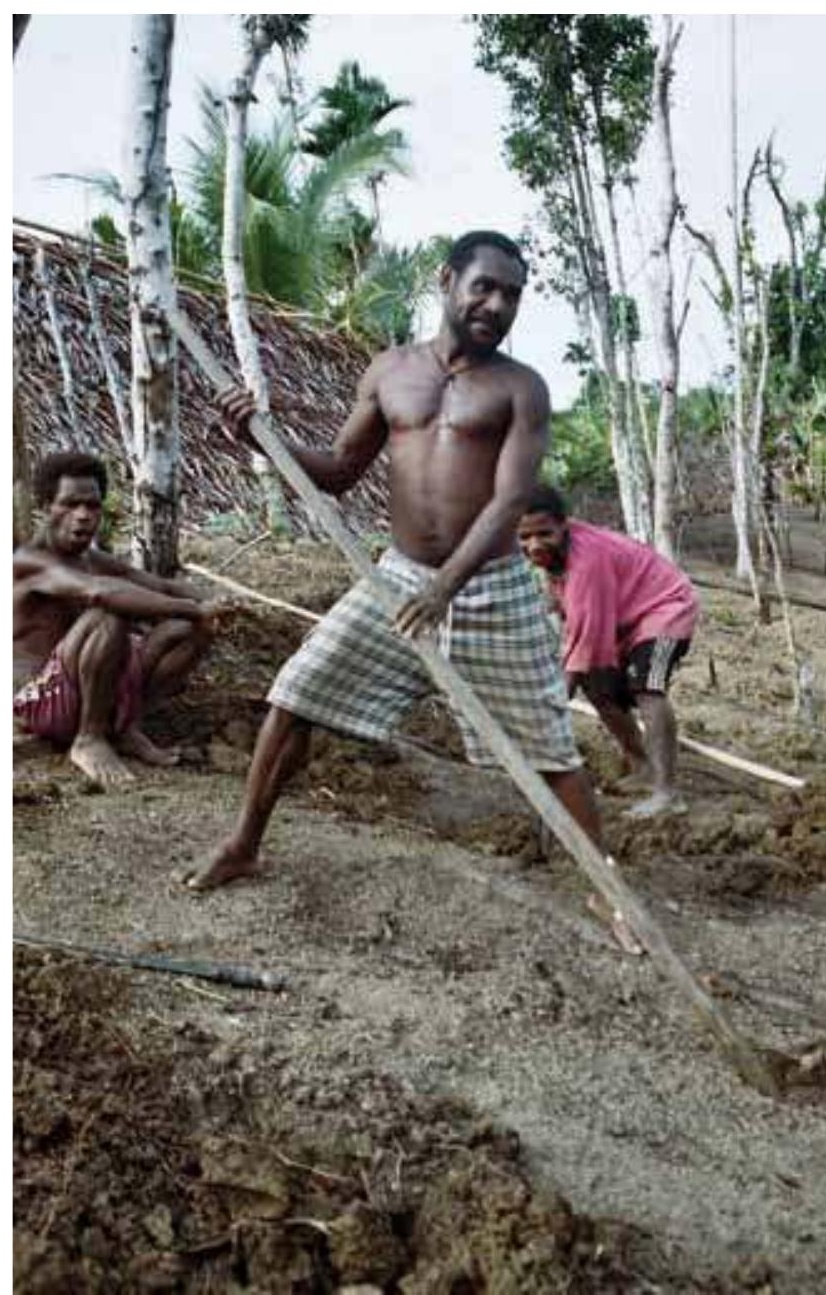




\section{Processus de matérialisation}

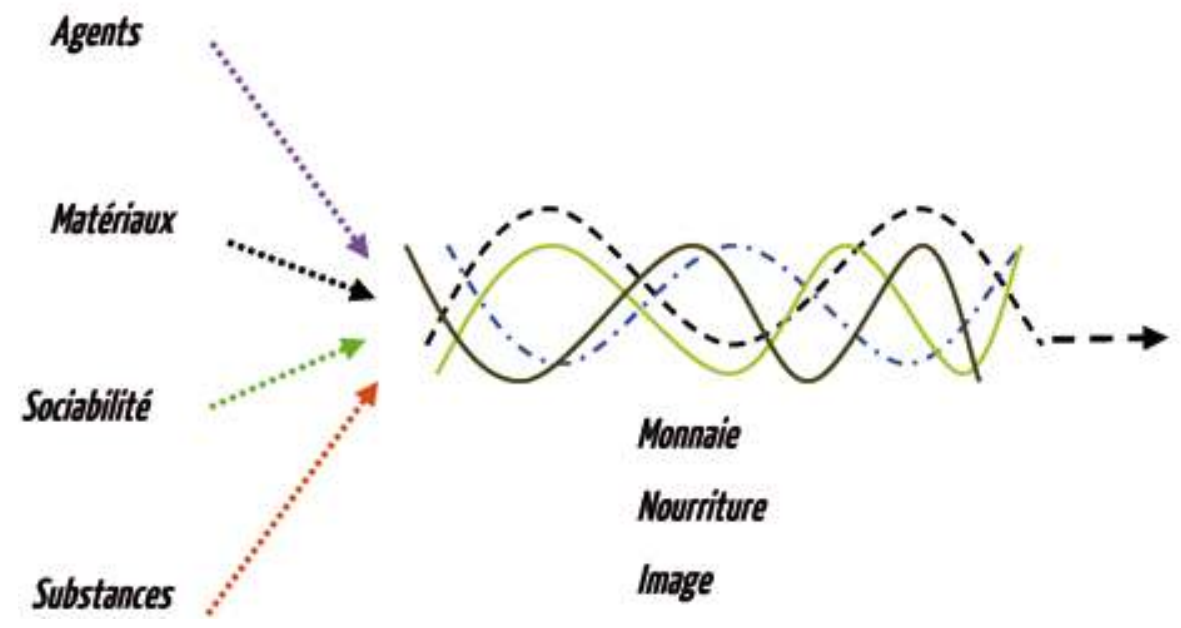

\section{NOTES}

Toutes les photos sont de l'auteur, sauf mention contraire. Photo d'ouverture: plusieurs variétés d'ignames (petites et grandes), tabac, noix d'arec (sur le côté on devine également des anneaux de coquillages et argent) rassemblés pour une compensation matrimoniale. (mars 2002).

1. Les ignames, comme d'autres tubercules, se propagent par reproduction végétative, chaque morceau de tubercule pouvant donner plusieurs clones de l'individu original (cf. Haudricourt 1964).

2. Parfois traduit sous le terme d'agence (Descola 2007) ou d'agentivité (Gell 2008)

3. J'ai détaillé ailleurs, la chaîne opératoire de la production d'ignames, cf. Coupaye 2009a, 2009b.

4. Chaque cultivateur s'occupe de quatre à cinq jardins: un jardin de grandes ignames, isolé, personnel, à l'accès restreint; un jardin de petites ignames ouvert dans l'année en cours; un jardin ouvert l'année précédente; enfin, des jardins ouverts anciennement qui servent de réservoirs de semences pour les jardins plus récents. À ceci, il faut ajouter, bien souvent, un jardin de culture de rente (café, cacao ou vanille). Comprendre le rôle des grandes ignames implique donc d'intégrer à la chaîne opératoire les actions entreprises dans ces différents jardins, ainsi que leurs temporalités.

5. Bien qu'un cultivateur plante généralement deux ou trois grandes ignames dans son jardin.

6. Ou comme série d'étapes incluant le rassemblement des ressources nécessaire à la bonne réception des invités, la fabrication et les échanges permettant d'obtenir les ornements, les relations entre villages, etc. 


\section{RÉFÉRENCES}

Bloch, Maurice, 1999, « Une Nouvelle théorie de l'art: À propos d'Art and Agency d'Alfred Gell », Terrain $n^{\circ} 32: 119-28$.

Coupaye, Ludovic, 2009 a, « What's the Matter with Technology? Long (and Short) Yams Materialisation and Technology in Nyamikum village, Maprik district, East Papua New Guinea », The Australian Journal of Anthropology 20: 93-111.

Coupaye, Ludovic, 2009 b, «Ways of Enchanting: Yam cultivation and Display in Nyamikum Village, Maprik, PNG », Journal of Material Culture 14 (4) : 433-458.

Cresswell, Robert, 1996, Prométhée ou Pandore? Propos de technologie culturelle. Paris, Éditions Kimé.

Descola, Philippe, 2007, « Passages de témoins », Le Débat: Le Moment du Quai Branly, 147: 136-153.

Forge, Anthony, 1962, «La Peinture, substance magique », Palette, 9:9-16.

Forge, Anthony, 1973, "Style and Meaning in Sepik Art », in Primitive Art And Society, Anthony Forge (ed.) Londres \& Oxford, Oxford University Press, Ely House: 169-92.

Gell, Alfred, 1992, « The Technology of Enchantment and the Enchantment of Technology », in Anthropology Art and Aesthetics, Jeremy Coote et Anthony Shelton eds., Oxford, Clarendon Press: 40-63.

Gibson J. J. 1977, « The Theory of Affordances », in Perceiving, Acting and Knowing, R. Shaw \& J. Bransford eds., Hillsdale, NJ, Erlbaum: 67-82.

Haudricourt, André-Georges, 1987 [1964], « Nature et Culture dans la civilisation de l'igname: l'origine des clones et des clans", in La Technologie, science humaine, Recherches d'ethnologie et d'histoire des techniques. Paris, Maison des sciences de l'homme: 287-98.

Huber-Greub, Barbara, 1988, Kokospalmenmenschen: Boden und Alltag und ihre Bedeutung im Selbstverständnis der Abelam von Kimbangwa (East Sepik Province, Papua New Guinea). Basler Beiträger zur Ethnologie, Band 27. Basel, Weipf et Co, AG Verlag.

Ingold, Tim, 2007, « Materials A gainst Materiality ». Archaeological Diaologues, 14 (1) : 1-16.

Jeudy-Ballini, Monique and Bernard Juillerat eds., 2002, People and Things. Social Mediation in Oceania. Durham (NC), Carolina Academic Press.

Kaberry, Phyllis, 1941, « The Abelam Tribe, Sepik District. New Guinea », A Preliminary report, Oceania, 11 (3) : 233-58; (4) : 345-67.

Keane, Webb, 2005, « Signs Are Not the Garb of Meaning: On the Social Analysis of Material Things » in Daniel Miller ed., Materiality. Durham et Londres, Duke University Press : 182-205.

Kopytoff, Igor. 1986. « The Cultural Biography of Things: Commoditization as Process. in Arjun Appadurai ed., The Social Life of Things: Commodities in Cultural Perspective. Cambridge, Cambridge University Press: 65-91.

Lara (de), Philippe, 2005, Le Rite et la raison. Wittgenstein anthropologue. Paris, Ellipses.

Latour, Bruno, 1991, Nous n'avons jamais été Modernes: Essai d'anthropologie symétrique. Paris, La Découverte.

Lea, David A. M., 1964, Abelam land and sustenance horticulture in an area of high population density, Maprik, New Guinea, Canberra, Australian National University [thèse de doctorat].

Mauss, Marcel, 1950 b (1935), « Les Techniques du corps », in Sociologie et anthropologie. Paris, PUF : 365386.

Mauss, Marcel, 1968 [1909], Euvres. Tome I: Les Fonctions sociales du sacré. Paris, Éditions de Minuit: 357-477.

Miller, Daniel ed., 2005, Materiality. Durham \& Londres, Duke University Press.

Rubel, Paul G., \& Abraham Roseman, 1978, Your Own Pigs You May Not Eat: A Comparative Study of New Guinea Societies. Chicago et Londres, The University of Chicago Press.

Strathern, Marilyn, 1999, Property, Substance and Effect: Anthropological Essays on Persons and Things. Londres et New Brunswick (NJ), The Athlone Press.

Tuzin, Donald, 1972, "Yam symbolism in the Sepik: an Interpretation Account », Southwestern Journal of Anthropology, xxviII: 230-54. 
Wagner, Roy, 1986 [1975], Symbols that Stand for Themselves. Chicago \& Londres, University of Chicago Press.

Wagner, Roy, 1991, « The Fractal Person », in Maurice Godelier et Marilyn Strathern (eds.), Big Men \& Great Men: Personifications of Power in Melanesia, . Cambridge, Cambridge University Press; Paris, Éditions de la Maison des sciences de l'homme: 159-73.

Warnier, Jean-Pierre, 2001, «A Praxeological Approach to Subjectivation in a Material World », in Journal of Material Culture, 6 (1): 5-24.

Wittgenstein, Ludwig, 1993 [1922], Tractatus Logico-Philosophicus. Paris, Gallimard.

\section{RÉSUMÉ}

Décrire des objets hybrides. Les grandes ignames décorées du village de Nyamikum, district de Maprik, province de l'Est Sepik, Papouasie-Nouvelle-Guinée. Dans ce court article, Ludovic Coupaye présente de manière synthétique un usage élargi de la notion de chaîne opératoire appliquée à un objet original, les grandes ignames décorées de la province du Sépik occidental, en Papouasie Nouvelle Guinée. L'objectif principal est d'illustrer quelques-uns des éléments théoriques et méthodologiques issus des approches francophones et anglophones de l'anthropologie de l'art et de la culture matérielle. Il commence par démontrer comment, par la méthodologie de la chaîne opératoire, il devient possible de comprendre le rôle multivalent d'images d'ancêtres, de monnaies cérémonielles et de nourriture des tubercules décorés. Puis il relie brièvement les éléments mis au jour par la méthodologie à des concepts récents utilisés dans l'anthropologie de la culture matérielle et de l'anthropologie de la Mélanésie, tels que la matérialité, la notion d'objet hybrides ou encore l'agency de l'art.

\section{ABSTRACT}

How to Describe Hybrid Objects. Large Inams Decorated with the Nyamikum Village, District of Maprik, Province of the East Sepik, Papouasie- News-Guinea. In this brief paper, Coupaye presents his utilisation of the notion of chaîne opératoire (operational sequence) in an extended way, to analyse the decorated long yams of the East sepik Province in Papua New Guinea. The aim is to illustrate some of the theoretical and methodological elements used in Francophones and Anglophones anthropology of art and material culture. Coupaye starts by demonstrating how, by using the chaîne opératoire, it is possible to understand the multivalent role of decorated tubers as ancestors images, valuables and food. Then he relates the elements revelead by such methodology to recent concepts used in anthropology of material culture and Mélanésian ethnography, such as materiality, the notion of « hybrid objets», or agency of art.

\section{MOTS CLÉS}

Chaîne opératoire, anthropologie de l'art, anthropologie des techniques, agency, materialité, methodology.

\section{KEYWORDS}

Operational sequence (Chaîne opératoire), anthropology of art, anthropology of techniques, agency, materiality, methodology. 